\title{
Interference Alignment in Partially Connected Interfering Multiple-Access and Broadcast Channels
}

\author{
Maxime Guillaud \\ Vienna University of Technology, \\ Gußhausstraße 25/389 \\ A-1040 Vienna, Austria \\ e-mail: guillaudetuwien.ac.at
}

\author{
David Gesbert \\ EURECOM \\ 2229 route des crêtes, BP 193, \\ F-06560 Sophia-Antipolis cedex, France \\ e-mail: David.Gesbert@eurecom. fr
}

\begin{abstract}
We consider interference alignment (IA) in the Linterfering multiple access channels (MACs) network, a particular case of the partially connected interfering MACs network whereby the number of interference links per MAC is bounded regardless of the total number of MACs in the network. Conversely to the fully-connected case, we show that interference alignment can be achievable in a network of arbitrary size, while the per-user signaling dimension remains bounded. We provide necessary conditions for the feasibility of IA, discuss their sufficiency, and introduce an algorithm capable of providing practical solutions. These results also apply to the dual case of partially connected interfering broadcast channels (IBCs), and have practical applications to both the uplink and downlink of large cellular networks.
\end{abstract}

\section{INTRODUCTION}

Interference alignment (IA) has been introduced in [1] for the case of the K-user MIMO interference channel (IC) with constant coefficients. In that context, when the number of antennas at each node is bounded, IA is only achievable among a finite number of users [2]. Several attempts at applying IA to more general models departing from the classical Kuser IC model, can be found in the literature. In [3], IA is applied to multiple interfering Multiple-Access Channels (MAC). However the method relies on a non-trivial channel model (called decomposable), and is not generalizable to general MIMO Gaussian channels. Interfering MACs (IMACs) are also investigated in [4], but the results are limited to the twoMACs case. The model of interfering broadcast channels (BC) is considered in [5]. Considering only two cells, they provide an achievable scheme whereby interference is aligned between out-of-cell and intra-cell interference. Interfering multipleinput single-output (MISO) BCs with time-varying channel coefficients have been considered in [6], where their degrees of freedom are analyzed and bounded.

All these results have in common the fact that they only apply to a finite number of cells or links because of the finite number of degrees of freedom offered by the spatial dimension at the transmitter and at the receiver. Indeed, there exists a few approaches to allow for a scaling of IA across a growing number of links or users: (i) let the number of antennas per user grow arbitrarily large with the size of the network, (ii) apply a power control scheme, which effectively reduces the number of active nodes to preserve the feasibility of IA over the network, (iii) align some of the interference, and treat the rest as (colored) noise, or (iv) assume a network-wise channel model exhibiting a limited connectivity between the various nodes. Although interesting from an analytical point of view, approach (i) is not realistic in practice. Approach (ii) is more practical and was considered e.g. in [7], nevertheless, power control results in deactivating certain links and goes at the expense of the total multiplexing gain of the network. Approach (iii) was considered in [8], although its performance in large networks is unclear. This paper considers the situation (iv) in which natural attenuation effects (path loss, fading) cause the (at least partial) loss of connectivity between certain receivers and interfering transmitters. This scenario arises for instance in the cellular network context where the distance between non-neighboring cells causes a strong attenuation on far-away interference signals. On-off models are typically considered in order approximate this situation, in which each receiver is assumed to be receiving non-zero interference from a bounded set of transmitters only. This model is referred to as partially connected in the following.

Partially connected interference networks have been considered in [9], where the IC with time-varying channels is considered. [10] introduced the many-to-one interference channel, whereby only one user faces interference. The partially connected MIMO IC has been considered in [11], where an achievable scheme is proposed for certain problem dimensions.

This paper is a generalization of our previous result on the partially connected IC [12] to the case of the interfering BCs and MACs. The key contribution of this work is to show that, under mild conditions on the connectivity of the interference links in the model, IA is feasible among an arbitrary number of users while keeping the signaling dimension bounded. Specifically,

- We introduce a particular case of the partially connected MIMO interfering MACs network, the L-interfering MACs network, whereby the number of interfering links per transmitter and receiver is bounded. This represents a realistic assumption in the context of cellular networks with more than one user per cell.

- We provide a set of necessary conditions for the feasibility of IA in the particular case of the L-interfering MACs (and the dual L-interfering BCs). We discuss the 
sufficiency of these conditions.

- We show that interference can be ideally cancelled from IA in an L-interfering network of arbitrary size, while the number of antennas per user remains bounded.

- We adapt the algorithm from [14] for computing precoding matrices and receiver beamforming vectors that realize IA in our considered scenario.

As an example of potential application of our result in the context of cellular networks, we show that, if all base stations are equipped with 7 antennas, all users with 2 antennas each, and each cell receives interference from up to 3 other cells, then 2 users per cell can simultaneously achieve $1 \mathrm{DoF}$ to the base station while having the out-of-cell interference completely suppressed through IA. This holds regardless of the total number of cells in the network, and over constant MIMO channels. Note that this schemes achieves half of the DoF per user available without inter-cell interference over the same channel. Note also that this result does not rely on forming clusters of base stations, but rather aims at a joint solution of IA across a complete network of arbitrary number of cells.

\section{Partially Connected Interfering MiMO MACs}

Let us introduce the channel model underlying our work, i.e. the partially connected interfering MIMO MACs (PCI-MIMOMAC). We consider symmetric systems, whereby all involved MACs have the same dimension, namely $\mathrm{M}$ antennas at the receiver and $\mathrm{N}$ antennas at each of the $P$ transmitters, and consider a network comprised of $K$ such MACs (see Fig. 1).

For $k \in\{1, \ldots, K\}$, we write the $M$-dimensional signal received at the $k$-th MAC as

$$
\mathbf{y}^{(k)}=\sum_{p=1}^{P} \mathbf{H}_{p}^{(k, k)} \mathbf{x}_{p}^{(k)}+\sum_{l \in \mathrm{I}(k)} \sum_{p=1}^{P} \mathbf{H}_{p}^{(k, l)} \mathbf{x}_{p}^{(l)},
$$

where $\mathbf{x}_{p}^{(l)}$ denotes the $N$-dimensional vector signal from the $p$-th transmitter in the $l$-th MAC, and $\mathbf{H}_{p}^{(k, l)}$ is the $M \times N$ matrix channel from this transmitter to the receiver of the $k$-th MAC. The first term in (1) represents the superposition of signals from users in the considered MAC, while the second term accounts from interference from the other MACs. $\mathrm{I}(k) \subset\{1, \ldots, k-1, k+1, \ldots, K\}$ denotes the set of MACs which interfere with the $k$-th MAC (for the sake of simplicity, we assume that either all or none of the transmitters in a given MAC can be heard by the receiver of the $k$-th MAC). We also let $\mathrm{I}^{-1}(l)=\{k \mid l \in I(k)\}$ for $l \in\{1, \ldots, K\}$, i.e. $\mathrm{I}^{-1}(l)$ is the set of MACs which are affected by interference from the transmitters of MAC $l$. In the example pictured in Fig. $1, \mathrm{I}(1)=\{2,3\}$ while $\mathrm{I}^{-1}(1)=\{2\}$.

Note that this model can be particularized in several ways:

- By letting $\mathrm{I}(k)=\{1, \ldots, k-1, k+1, \ldots, K\}$, one obtains the case of $K$ fully connected interfering MACs.

- By letting $\mathrm{I}(k)=\{k-1, k+1\} \quad \forall k \in\{2, \ldots, K-1\}$, we obtain the classical Wyner model.

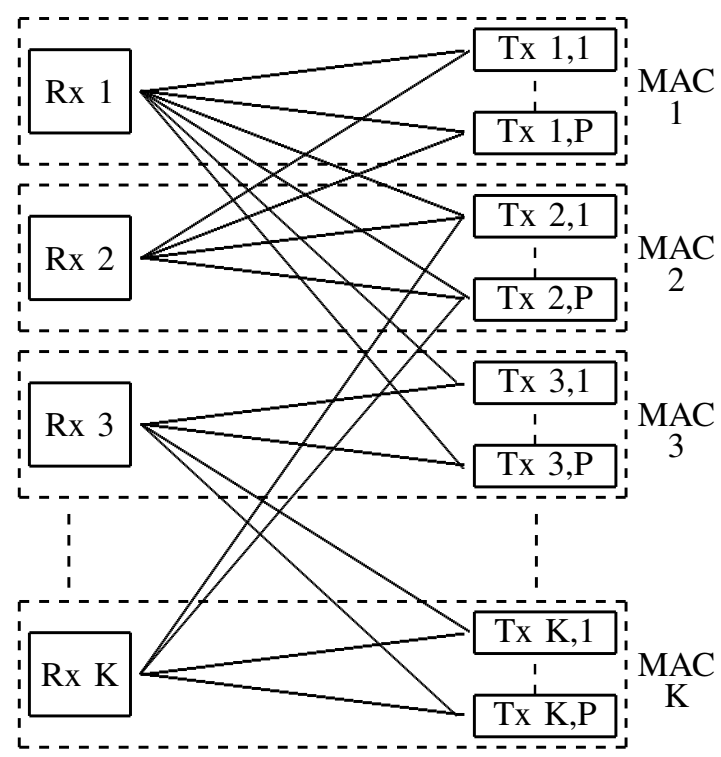

Fig. 1. Example connectivity graph in a partially connected interfering MACs model. Lines represent channels with non-zero coefficients.

- By letting $P=1$, we obtain the partially connected MIMO IC.

We now introduce another particularization of this model, which we will use in the sequel.

Definition 1 (L-interfering MACs network): The network formed by the $K$ partially connected MIMO MACs as defined in eq. (1) is $L$-interfering for some $L<K$, iff

$$
\forall k \in\{1, \ldots, K\}, \quad|\mathrm{I}(k)| \leq L \quad \text { and } \quad\left|\mathrm{I}^{-1}(k)\right| \leq L,
$$

where $|\cdot|$ denotes the cardinality operator.

Intuitively, Definition 1 corresponds to the case where the number of MACs interfering with the receiver of any MAC in the network, and the number of MACs whose receivers get interference from a given transmitter, are bounded by some value $L$. We deem this model more realistic than e.g. the Wyner model, since it can correctly represent the interference situation encountered in a cellular model with regular cell arrangement and inter-cell interference limited to a fixed radius, while the (single-dimensional) Wyner model can not faithfully replicate the connectivity situation encountered in such a 2-dimensional network.

\section{IA OVER THE PCI-MIMO-MAC NETWORK}

We are now concerned with achieving IA in the PCI-MIMOMAC network introduced in the previous section.

\section{A. Definition}

Assume that the signal transmitted by user $p$ in the $l$-th MAC is linearly precoded by the $N \times D$ full column rank matrix $\mathbf{V}_{p}^{(l)}$, i.e. $\mathbf{x}_{p}^{(l)}=\mathbf{V}_{p}^{(l)} \mathbf{s}_{p}^{(l)}$ where $\mathbf{s}_{p}^{(l)}$ is a vector with $D \leq N$ coefficients.

We wish all interference at the receiver of a given MAC $k$ in the network, coming from transmitters in other MACs to be 
restricted to a subspace of dimension $M-D^{\prime}$, where $D^{\prime} \leq M$ denotes the dimension of the interference-free subspace ${ }^{1}$. This is possible iff we can find full column rank $M \times D^{\prime}$ projection matrices $\mathbf{U}^{(k)}, k=1, \ldots, K$ which suppresses all interference, i.e. such that $\mathbf{U}^{(k)}{ }^{H} \sum_{l \in \mathrm{I}(k)} \sum_{p=1}^{P} \mathbf{H}_{p}^{(k, l)} \mathbf{V}_{p}^{(l)} \mathbf{s}_{p}^{(l)}=$ $0 \forall k$. Intuitively, in the cellular framework, this corresponds to aligning all out-of-cell interference into a subspace of codimension $D^{\prime}$ at each receiver (it is clear from (1) that, if this condition is fulfilled, the interference term in $\mathbf{U}^{(k)}{ }^{H} \mathbf{y}^{(k)}$ vanishes and one obtains an equivalent MAC channel with $D^{\prime}$ receive antennas and $P$ users with $D$ antennas each). Since the above must be true for all possible values of the transmitted symbols $\mathbf{s}_{p}^{(l)}$, this yields the following definition of IA over the PCI-MIMO-MAC:

Definition 2: IA with parameters $\left(D, D^{\prime}\right)$ is achieved in the PCI-MIMO-MAC iff there exist full column-rank $M \times D^{\prime}$ matrices $\mathbf{U}^{(k)}, k=1, \ldots, K$, and $N \times D$ matrices $\mathbf{V}_{p}^{(k)}$, $k=1, \ldots, K, p=1, \ldots, P$, s.t.

$$
\begin{aligned}
\mathbf{U}^{(k)}{ }^{H} \mathbf{H}_{p}^{(k, l)} \mathbf{V}_{p}^{(l)} & =0, \\
\forall k \in\{1, \ldots, K\}, \quad p & \in\{1, \ldots, P\}, \quad l \in \mathrm{I}(k) .
\end{aligned}
$$

Remark 1 (Partially Connected MIMO Interfering BCs): The case of $K$ partially connected MIMO interfering BCs (PC-MIMO-IBC) with $N \times M$ channels $\mathbf{H}_{p}^{(k, l)}{ }^{H}$, with inputoutput relationship $\mathbf{x}_{p}^{(l)}=\mathbf{H}_{p}^{(l, l)} \mathbf{y}^{(l)}+\sum_{k \in \mathrm{I}^{-1}(l)} \mathbf{H}_{p}^{(k, l)}{ }^{H} \mathbf{y}^{(k)}$, is dual of the interfering MAC case, as far as IA is concerned. Using the $M \times D^{\prime}$ linear precoder $\mathbf{U}^{(k)}$ at transmitter $k$, and the interference suppression matrix $\mathbf{V}_{p}^{(l)}$ at receiver $p$ in the $k$ th BC, it is clear that IA is achieved iff $\mathbf{V}_{p}^{(l)}{ }^{H} \mathbf{H}_{p}^{(k, l)}{ }^{H} \mathbf{U}^{(k)}=$ $0 \forall l \in\{1, \ldots, K\}, p \in\{1, \ldots, P\}, k \in \mathrm{I}^{-1}(l)$, which is equivalent to condition (3) up to the exchange of $\mathrm{I}_{\text {for }} \mathrm{I}^{-1}$, which leaves (2) unchanged. Therefore, Definition 1 extends immediately to networks of interfering BCs. Henceforth, we will focus on L-interfering MIMO-MAC networks, bearing in mind that our conclusions apply to the dual L-interfering MIMO-BC networks as well.

\section{B. Achievable DoF with IA}

If IA according to Definition 2 is achieved, zero-forcing of the interference at receiver $k$ yields

$$
\begin{gathered}
\widetilde{\mathbf{y}}^{(k)}=\mathbf{U}^{(k)}{ }^{H} \mathbf{y}^{(k)}=\mathbf{U}^{(k)}{ }^{H} \sum_{p=1}^{P} \mathbf{H}_{p}^{(k, k)} \mathbf{V}_{p}^{(k)} \mathbf{s}_{p}^{(k)} \\
=\underbrace{\mathbf{U}^{(k)^{H}}\left[\mathbf{H}_{1}^{(k, k)} \ldots \mathbf{H}_{P}^{(k, k)}\right] \operatorname{diag}\left(\mathbf{V}_{1}^{(k)}, \ldots, \mathbf{V}_{P}^{(k)}\right)}_{\mathbf{H}^{(k)}}\left[\begin{array}{c}
\mathbf{s}_{1}^{(k)} \\
\vdots \\
\mathbf{s}_{P}^{(k)}
\end{array}\right]
\end{gathered}
$$

Since both $\mathbf{U}^{(k)}$ and $\operatorname{diag}\left(\mathbf{V}_{1}^{(k)}, \ldots, \mathbf{V}_{P}^{(k)}\right)$ are full columnrank, under mild assumptions on the distribution of the channel coefficients (for instance, if they are drawn i.i.d. from a continuous distribution, see [1]), we have that $\operatorname{rank}\left(\mathbf{H}^{(k)}\right)=$

\footnotetext{
${ }^{1}$ We give further comments on the proper choice for $D^{\prime}$ in Section III-B.
}

$\min \left(D^{\prime}, P D\right)$ almost surely (a.s.). Therefore, the resulting achievable DoF per MAC is equal to $\min \left(D^{\prime}, P D\right)$. Clearly, choosing $D^{\prime}=D P$ is the optimal choice if one seeks to maximize the total DoF achieved by IA in the network. In cases where DoF maximization is not the sole objective, choosing $D^{\prime}>D P$ might be desirable [13].

\section{Iterative Algorithm}

The following algorithm is adapted from the one in [14]. Although no proof of its optimality is available, algorithms of this class have been found by us and other authors to reliably converge to an IA solution whenever one exists.

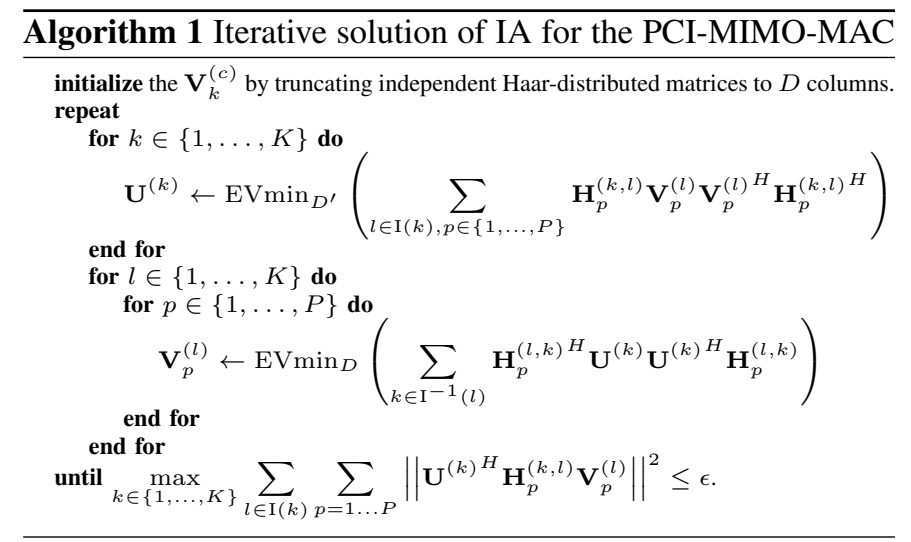

The $\operatorname{EVmin}_{n}(\cdot)$ operator denotes the selection of the eigenvectors associated with the $n$ lowest magnitude eigenvalues of the argument matrix. $\epsilon$ is a small value below which the sum of the interference powers in each MAC is deemed negligible.

Remark 2: Note that for any $k \in\{1, \ldots, K\}$, the algorithm above only requires channel and precoding/interference suppression matrix information for the MACs indexed by $\mathrm{I}(k)$ and $\mathrm{I}^{-1}(k)$. Indeed, in the presence of reciprocal channels (as in [14]), the algorithm is naturally distributed, and the argument in the second EVmin has a physical meaning. Note however that, since Algorithm 1 merely solves (3), it can also be used in a centralized manner to achieve IA over nonreciprocal channel. Note also that its global complexity per iteration scales linearly with $K$, i.e. the complexity per MAC per iteration remains constant, regardless of the size of the network.

\section{AChievability NeCessary Condition}

Our analysis is based on the technique introduced in [2] for the case of the (fully connected) MIMO IC, and relies similarly on the notion of proper system of equations applied to (3). A system of polynomial equation is identified as proper iff, for any subset of the equations, the number of variables involved is at least as large as the number of equations (see the discussion in [2]). Properness is a necessary condition for IA to be feasible in a network, although it is in general not a sufficient condition (see Section IV-A). We now characterize which of the L-interfering MIMO-MACs networks are proper. This is the object of the following theorem. 
Theorem 1: A sufficient condition for the system of equation (3) to be proper for the case of an L-interfering MIMO MACs network (Definition 1) is that

$$
P D(N-D)+D^{\prime}\left(M-D^{\prime}\right)-D D^{\prime} P L \geq 0 .
$$

Furthermore, (5) is also a necessary condition if $\forall k \in\{1, \ldots, K\},|\mathrm{I}(k)|=L$.

Note that (5) is independent from the total number $K$ of MACs in the network. Intuitively, this is because the constraint in (2) ensures that the number of scalar equations involved in the system of equations (3) (or any subset thereof) scales linearly with $K$ instead of quadratically for the fully connected MIMO IC where $|\mathrm{I}(k)|=K-1 \forall k$, and so does the number of variables. We now give a formal proof of Theorem 1 .

Proof:

In a first step, we prove that (5) is necessary in the case $|\mathrm{I}(k)|=L \forall k$. For this, we consider the total number of equations and variables involved in (3). The number of distinct tuples $(k, l, p)$ involved in (3) is trivially $K L P$, and each of the matrix equalities represents $D^{\prime} \times D$ scalar equations. This yields a total of $N_{e}=K L P D D^{\prime}$ equations. The number of variables in the $\mathbf{V}_{p}^{(l)}$ and $\mathbf{U}^{(k)}$ matrices must be counted while paying attention to the fact that multiple parameterizations of the same choice of a subspace are possible, and must be counted only once. As shown in [2], each $\mathbf{V}_{p}^{(l)}$ must be counted as $D(N-D)$ variables, while each $\mathbf{U}^{(k)}$ represents $D^{\prime}\left(M-D^{\prime}\right)$ variables. Therefore, we have $N_{v}=K P D(N-$ $D)+K D^{\prime}\left(M-D^{\prime}\right)$. Notice now that if (5) is not fulfilled, we have immediately that $N_{v}<N_{e}$, i.e. the system is not proper. Therefore, (5) is necessary.

We now prove the sufficient part. We need to check that the inequality between number of equations and variables is verified for all possible subsets of the equations. Let us introduce some formalism. Let $\mathrm{S}=$ $\left\{\left(d^{\prime}, k, l, p, d\right) \in\left\{1, \ldots, D^{\prime}\right\} \times\{1, \ldots, K\} \times\{1, \ldots, K\}\right.$ $\times\{1, \ldots, P\} \times\{1, \ldots, D\}$ s.t. $l \in \mathrm{I}(k)\}$. Each tuple in $\mathrm{S}$ corresponds to one scalar IA equation from eq. (3). Let A $\subset S$ an arbitrary subset of S. Let $N_{v}^{\mathrm{A}}$ denote the number of variables involved in any of the equations designated by $\mathrm{A}$, and $N_{e}^{\mathrm{A}}=|\mathrm{A}|$ the number of those equations. We need to prove that $N_{v}^{\mathrm{A}} \geq N_{e}^{\mathrm{A}}$.

We need the following definitions:

$$
\begin{aligned}
\overline{\mathrm{K}} & =\left\{k \text { s.t. } \exists\left(d^{\prime}, l, p, d\right) \text { s.t. }\left(d^{\prime}, k, l, p, d\right) \in A\right\} \\
\overline{\mathrm{LP}} & =\left\{(l, p) \text { s.t. } \exists\left(d^{\prime}, k, d\right) \text { s.t. }\left(d^{\prime}, k, l, p, d\right) \in A\right\} \\
\overline{\mathrm{KL}} & =\left\{(k, l) \text { s.t. } \exists\left(d^{\prime}, p, d\right) \text { s.t. }\left(d^{\prime}, k, l, p, d\right) \in A\right\} \\
\overline{\mathrm{KLP}} & =\left\{(k, l, p) \text { s.t. } \exists\left(d^{\prime}, d\right) \text { s.t. }\left(d^{\prime}, k, l, p, d\right) \in A\right\} \\
\overline{\mathrm{D}}(l, p) & =\left\{d \text { s.t. } \exists\left(d^{\prime}, k\right) \text { s.t. }\left(d^{\prime}, k, l, p, d\right) \in A\right\} \\
\overline{\mathrm{D}^{\prime}}(k) & =\left\{d^{\prime} \text { s.t. } \exists(l, p, d) \text { s.t. }\left(d^{\prime}, k, l, p, d\right) \in A\right\}
\end{aligned}
$$

Intuitively, $\overline{\mathrm{K}}$ is the set of indices $c$ which appear in at least one tuple in $\mathrm{A} ; \overline{\mathrm{D}}(l, p)$ is the set of indices $d$ which appear in at least one tuple in A together with a given $(l, p)$; etc.

Using these definitions, the number of variables involved in the beamformer at transmitter $p$ in MAC $l$ is
$|\overline{\mathrm{D}}(l, p)|(N-|\overline{\mathrm{D}}(l)|)$, while the number of variables involved in the projection filter at receiver $k$ is $\left|\overline{\mathrm{D}^{\prime}}(k)\right|\left(M-\left|\overline{\mathrm{D}^{\prime}}(k)\right|\right)$. We have therefore

$$
\begin{aligned}
& N_{v}^{\mathrm{A}}= \sum_{k \in \overline{\mathrm{K}}}\left|\overline{\mathrm{D}^{\prime}}(k)\right|\left(M-\left|\overline{\mathrm{D}^{\prime}}(k)\right|\right) \\
& \quad+\sum_{(l, p) \in \overline{\mathrm{LP}}}|\overline{\mathrm{D}}(l, p)|(N-|\overline{\mathrm{D}}(l, p)|) \\
& \geq \sum_{k \in \overline{\mathrm{K}}}\left|\overline{\mathrm{D}^{\prime}}(k)\right|\left(M-D^{\prime}\right)+\sum_{(l, p) \in \overline{\mathrm{LP}}}|\overline{\mathrm{D}}(l, p)|(N-D)(13)
\end{aligned}
$$

since the cardinalities of $\overline{\mathrm{D}}(l)$ and $\overline{\mathrm{D}^{\prime}}(k)$ are upper bounded respectively by $D$ and $D^{\prime}$ by definition of the sets.

Let us now fix $k, l$ and $p$, and consider the tuples $\left(d^{\prime}, k, l, p, d\right)$ that appear in A. Clearly there are at most $|\overline{\mathrm{D}}(l, p)|\left|\overline{\mathrm{D}^{\prime}}(k)\right|$ such tuples. Therefore, summing over all possible $(k, l, p)$,

$$
|\mathrm{A}| \leq \sum_{(k, l, p) \in \overline{\mathrm{KLP}}}|\overline{\mathrm{D}}(l, p)|\left|\overline{\mathrm{D}^{\prime}}(k)\right| .
$$

Since $\left|\overline{\mathrm{D}^{\prime}}(k)\right| \leq D^{\prime} \forall k$, we have

$$
\begin{aligned}
|\mathrm{A}| & \leq \sum_{(k, l, p) \in \overline{\mathrm{KLP}}}|\overline{\mathrm{D}}(l, p)| D^{\prime} \\
& \leq \sum_{(l, p) \in \overline{\mathrm{LP}}}\left|\mathrm{I}^{-1}(l)\right||\overline{\mathrm{D}}(l, p)| D^{\prime} \\
& \leq \sum_{(l, p) \in \overline{\mathrm{LP}}} L|\overline{\mathrm{D}}(l, p)| D^{\prime}
\end{aligned}
$$

where (16) stems from the fact that $(k, l, p) \in \overline{\mathrm{KLP}}$ implies $k \in \mathrm{I}^{-1}(l)$, and that $\mathrm{I}^{-1}(l)$ has at least as many elements as its restriction to those appearing in A. (17) stems directly from Definition 1. Similarly, starting again from eq. (14),

$$
\begin{aligned}
|\mathrm{A}| & \leq \sum_{(k, l, p) \in \overline{\mathrm{KLP}}} D\left|\overline{\mathrm{D}^{\prime}}(k)\right| \leq P \sum_{(k, l) \in \overline{\mathrm{KL}}} D\left|\overline{\mathrm{D}^{\prime}}(k)\right| \\
& \leq P \sum_{k \in \overline{\mathrm{K}}}|\mathrm{I}(k)| D\left|\overline{\mathrm{D}^{\prime}}(k)\right| \leq L P D \sum_{k \in \overline{\mathrm{K}}}\left|\overline{\mathrm{D}^{\prime}}(k)\right| .
\end{aligned}
$$

Combining (13), (17) and (19) yields

$$
N_{v}^{\mathrm{A}} \geq|\mathrm{A}|\left(\frac{M-D^{\prime}}{L P D}+\frac{N-D}{L D^{\prime}}\right) .
$$

Finally, (5) ensures that the second term in the right-hand side of (20) is greater or equal to 1 , yielding $N_{v}^{\mathrm{A}} \geq|\mathrm{A}|=N_{e}^{\mathrm{A}}$.

\section{A. Discussion about sufficiency}

As mentioned above, having a proper system of equations (3) is not sufficient to guarantee that IA according to Definition 2 is feasible. Indeed, taking the example from [2] of the $(3 \times 3,2)^{2}$ IC (which in our notations corresponds to $K=2$, $L=1, P=1, M=N=3, D=D^{\prime}=2$ ), eq. (5) is fulfilled, while IA is not feasible since it would otherwise violate a general bound on the achievable DoF [15], which we repeat here: the total DoF achievable over a two-user IC with respectively $M_{1}$ and $M_{2}$ antennas at the receivers and 
$N_{1}, N_{2}$ antennas at the transmitters, is upper bounded by $\min \left(M_{1}+M_{2}, N_{1}+N_{2}, \max \left(M_{1}, N_{2}\right), \max \left(M_{2}, N_{1}\right)\right)$. In our example, this bound dictates that the total achievable DoF can not be higher than 3 , while $K D=4$.

Other bounds on the achievable DoF can be obtained by letting certain groups of transmitters and receivers collaborate (which does not reduce the achievable DoF) inside our IMAC network in order to create an IC. In particular, for any $k \in$ $\{1, \ldots, K\}$, we can let all transmitters and all receivers in a number $J \leq|I(k)|(\leq L)$ of MACs interfering with receiver $k$ cooperate among themselves. Applying the aforementioned bound to the resulting two-user IC with respectively $M$ and $J M$ receive antennas, and $P N$ and $J P N$ transmit antennas, and fixing $D^{\prime}=P D$ which maximizes the DoF achievable through IA, yields

$$
\begin{aligned}
(J+1) P D \leq \min & \{(J+1) M,(J+1) P N, \\
& \max (M, J P N), \max (J M, P N)\} .
\end{aligned}
$$

Condition (21) for $J \leq L$ rules out certain cases where IA is not achievable while the system is proper, although it still does not form a sufficient condition. For instance, exhaustive search (using Algorithm 1 to provide existence certificates) through $(P, M, N, L, D) \in$ $\{1, \ldots, 5\} \times\{1, \ldots, 20\} \times\{1, \ldots, 5\} \times\{1, \ldots, 8\} \times\{1, \ldots, 5\}$, with $K=15$, and $D^{\prime}=P D$ singled out the case $(P, L, M, N, D)=(2,1,5,5,2)$ (for arbitrary $K$ ) which is not ruled out by either (5) or (21), while it was found experimentally to be unfeasible.

Recent results concerning the sufficiency of the proper condition to achieve IA in the $K$-user (fully connected) MIMO IC have appeared shortly before the publication of this paper in [16] and [17]. Both results are based on algebraic geometry considerations. The case of a symmetric system with square channels $(M=N)$ and where each user wants to achieve $D$ DoF is considered in [17], where IA is shown to be feasible a.s. in proper systems with $K>3$ users. [16] shows that IA is feasible a.s. for proper systems with arbitrary number of antennas, provided that the number of antennas at each node is divisible by $D$.

We conjecture that this analysis can be generalized to the case of interfering MIMO MACs treated here, whenever the number of antennas at each node is divisible by $\mathrm{D}$, although the extension of the proofs is not immediate. Interestingly, the example cited above of the interfering MIMO MACs with parameters $(P, L, M, N, D)=(2,1,5,5,2)$ is one of the cases where an analysis of the type of [16] does not permit to conclude, since the number of antennas at the transmitters $(N=5)$ is not divisible by $D=2$.

\section{CONCLUSION}

We studied the feasibility of IA in the L-interfering MACs network, a model based on realistic assumptions regarding the locality of interference in large networks. The main contribution of the article is to highlight the existence of IA solutions in partially connected networks of potentially unbounded size, while the number of antennas per node can be kept finite. This is in sharp contrast with previous feasibility results obtained for IA in the fully-connected case, where at least one of the signaling dimensions had to grow unbounded with $K$. A set of necessary conditions was introduced, and their sufficiency was discussed.

\section{ACKNOWLEDGEMENTS}

M. Guillaud was supported in parts by the Austria Science Fund (FWF) through grant NFN SISE (S106), and by the FP7 HIATUS project of the European Commission (EC). D. Gesbert acknowledges funding from the EC's seventh framework programme FP7-ICT-2009 under grant agreement nr. 247223 (ARTIST4G). Both authors also acknowledge the support of the EC's FP7 NoE Newcom++.

\section{REFERENCES}

[1] V. R. Cadambe and S. A. Jafar, "Interference alignment and degrees of freedom of the K-user interference channel," IEEE Transactions on Information Theory, vol. 54, no. 8, pp. 3425-3441, Aug. 2008.

[2] C. M. Yetis, T. Gou, S. A. Jafar, and A. H. Kayran, "On feasibility of interference alignment in MIMO interference networks," IEEE Transactions on Signal Processing, vol. 58, no. 9, Sept. 2010.

[3] C. Suh and D. Tse, "Interference alignment for cellular networks," in Proc. Allerton Conference on Communications, Control and Computing, Monticello, IL, USA, Sept. 2008.

[4] J. Sun, Y. Liu, and G. Zhu, "On degrees of freedom of the cellular network," SCIENCE CHINA Information Sciences, vol. 53, pp. 1034 1043,2010

[5] C. Suh, M. Ho, and D. Tse, "Downlink interference alignment," IEEE Transactions on Information Theory, 2010, preprint from http://arxiv. org/abs/1003.3707.

[6] S.-H. Park and I. Lee, "Analysis of degrees of freedom of interfering MISO broadcast channels," in Proc. IEEE Global Telecommunications Conference (Globecom), Dec. 2009.

[7] Z. K. M. Ho, M. Kaynia, and D. Gesbert, "Distributed power control and beamforming on MIMO interference channels," in Proc. European Wireless Conference, Lucca, Italy, Apr. 2010.

[8] S. W. Peters and R. W. Heath, Jr., "Cooperative algorithms for MIMO interference channels," IEEE Transactions on Vehicular Technology, pp. 206-218, Jan. 2011.

[9] S. W. Choi and S.-Y. Chung, "On the multiplexing gain of K-user partially connected interference channel," 2008, submitted to IEEE Transactions on Information Theory, http://arxiv.org/abs/0806.4737.

[10] V. R. Cadambe and S. A. Jafar, "Interference alignment and a noisy interference regime for many-to-one interference channels," 2009, preprint from http://arxiv.org/abs/0912.3029.

[11] N. Lee, D. Park, and Y.-D. Kim, "Degrees of freedom on the Kuser MIMO interference channel with constant channel coefficients for downlink communications," in Proc. IEEE Global Telecommunications Conference (Globecom), Dec. 2009.

[12] M. Guillaud and D. Gesbert, "Interference alignment in the partially connected K-user MIMO interference channel," in Proc. European Signal Processing Conference (EUSIPCO), Barcelona, Spain, Aug. 2011.

[13] M. Guillaud, "Receive diversity and ergodic performance of interference alignment on the MIMO Gaussian interference channel," in Proc. Allerton Conference on Communication, Control, and Computing, Monticello, IL, USA, Sept. 2010.

[14] K. Gomadam, V. R. Cadambe, and S. A. Jafar, "Approaching the Capacity of Wireless Networks through Distributed Interference Alignment," IEEE Transactions on Information Theory, 2008, submitted. [Online]. Available: http://arxiv.org/abs/0803.3816

[15] S. A. Jafar and M. J. Fakhereddin, "Degrees of freedom for the MIMO interference channel," IEEE Transactions on Information Theory, vol. 53, no. 7, pp. 2637-2642, July 2007.

[16] M. Razaviyayn, G. Lyubeznik, and Z.-Q. Luo, "On the degrees of freedom achievable through interference alignment in a MIMO interference channel," Apr. 2011, preprint, http://arxiv.org/abs/1104.0992.

[17] G. Bresler, D. Cartwright, and D. Tse, "Settling the feasibility of interference alignment for the MIMO interference channel: the symmetric square case," Apr. 2011, preprint, http://arxiv.org/abs/1104.0888. 